\title{
La case des tout-petits au Sénégal
}

The "case des tout-petits" in Senegal

La "case des tout-petits" en Senegal

\section{Sophie Turpin Bassama}

\section{OpenEdition}

\section{Journals}

Édition électronique

URL : http://journals.openedition.org/ries/903

DOI : $10.4000 /$ ries.903

ISSN : 2261-4265

Éditeur

Centre international d'études pédagogiques

Édition imprimée

Date de publication : 1 avril 2010

Pagination : $65-75$

ISSN : 1254-4590

Référence électronique

Sophie Turpin Bassama, "La case des tout-petits au Sénégal », Revue internationale d'éducation de Sèvres [En ligne], 53 | avril 2010, mis en ligne le 01 avril 2013, consulté le 19 avril 2019. URL : http:// journals.openedition.org/ries/903; DOI : 10.4000/ries.903 


\section{La case des tout-petits au Sénégal}

\section{Sophie Turpin Bassama}

Le Sénégal compte 10425200 habitants, une population jeune et essentiellement rurale (58,5\%). Pays à faible revenu, il avait en 2005 un revenu national brut par tête (PPP RNB) de $\$ 1770$ et un taux de croissance du PIB de 5,1. Le secteur des services (63\% en 2005) est le plus gros contributeur au PIB. Il présente une grande disparité des revenus et de la consommation, avec un indice modéré de GINI de 41,3\% en 2007. Le taux de mortalité maternelle est relativement élevé. Une loi pour la parité des sexes vient d'être adoptée. Le système éducatif se structure à trois niveaux : formel, non formel et informel ${ }^{1}$. La « case des tout-petits» (CTP) dont il est question ici est logée au niveau formel. Dans l'énoncé de la politique nationale actuelle de développement intégré de la petite enfance, la CTP est le modèle choisi pour l'application du programme, elle est une référence pour les structures existantes.

\section{La Petite enfance, UNE PRIORITÉ POLITIQUE}

Depuis 2000, les autorités sénégalaises accordent une attention grandissante à la petite enfance, qui jouit d'un soutien politique fort et est devenue une priorité nationale grâce à une volonté politique portée par le président de la République et à un contexte international et régional favorable à l'éducation des jeunes enfants. De fait, la petite enfance a gagné en importance et en visibilité par l'adoption de différents traités clés relatifs aux droits des enfants ${ }^{2}$ insistant notamment sur la reconnaissance et le respect des principes universels en matière de droits à l'éducation et à la protection dès la naissance.

De la Déclaration de Jomtien en 1990, en passant par le Forum mondial sur l'éducation pour tous à Dakar, en avril 2000, et l'adoption des Objectifs $d u$ millénaire (OMD), le Sénégal, à l'instar de la communauté internationale, s'est engagé de manière résolue dans le développement de l'éducation pour tous (EPT) au plus tard à l'horizon 2015. Dans le cadre de l'initiative spéciale des Nations Unies pour l'Afrique, il s'est doté d'un plan décennal de l'éducation et de la formation afin d'atteindre les buts et objectifs de l'EPT qui, d'une certaine manière, intègre le développement de la petite enfance, la protection et l'éducation de

1. L'éducation non formelle comprend les centres d'alphabétisation, les écoles communautaires. L'éducation informelle regroupe les écoles de la rue, daaras, etc. L'éducation formelle inclut l'école maternelle. Pour une information plus complète voir Rayna, S. (2002).

2. La Convention des Nations Unies sur les droits de l'enfant de 1989 mais aussi la Charte africaine des droits et du bien-être de l'enfant (1990). 
cette dernière constituant son objectif premier. Ce plan, devenu le Programme de développement de l'éducation et de la formation (PDEF) établit le cadre de réalisation de la politique éducative du Sénégal. Le PDEF s'est fixé au départ trois objectifs : la démocratisation de l'accès à l'éducation de base, l'amélioration de la qualité des apprentissages et une gestion plus efficiente du système. Moyen sûr pour rompre le cercle vicieux de la pauvreté, la petite enfance figure, avec l'école élémentaire et l'alphabétisation des adultes, en bonne place parmi les priorités du PDEF.

Au cours des années quatre-vingts, période d'atonie de l'économie dans un contexte de forte croissance démographique, le système éducatif a subi le contrecoup des ajustements structurels et dû faire face à une grave crise à effets multiples : déficit en structures, en ressources humaines, matérielles et financières, et de surcroît mauvaise gestion du peu de moyens disponibles. Le système éducatif sénégalais a concentré alors ses efforts sur l'école élémentaire, au détriment de l'éducation préscolaire marginalisée.

Cette situation de crise a dévoilé les limites du secteur de l'éducation formelle et incité les pouvoirs publics, appuyés par les ONG, à se tourner vers le secteur de l'éducation non formelle qui développe des modèles peu coûteux, centrés sur une approche communautaire, et dont l'innovation de taille est l'implication des familles et des communautés considérées comme partenaires à part entière. C'est alors qu'une mutation est apparue dans la conception des programmes : des programmes de l'école maternelle classique fortement enracinés dans la tradition éducative où l'apprentissage est au cœur même de l'intervention éducative, on s'est orienté vers un modèle de programmes globaux associant l'éducation à une prise en charge sanitaire liée à la nutrition et à l'hygiène de l'enfant.

Vers la fin des années quatre-vingt-dix, une nouvelle vision de l'enfant et de son éducation fait évoluer les représentations des jeunes enfants et les modalités de leur prise en charge. L'éducation de la petite enfance bouge, se métamorphose : les politiques se réactualisent, les programmes et les pratiques se renouvellent en tentant de se nourrir à la fois de la tradition et de la modernité. Un nouveau paradigme pour l'éducation préscolaire se développe. Il remet en question le mode d'intervention dominant de l'école maternelle classique. Des structures plus visibles font désormais partie du décor et des stratégies innovantes les accompagnent. La case des tout-petits est au cour de cette mouvance.

\section{LA CASE DES TOUT-PETITS}

Le président de la République A. Wade, élu en 2000, manifeste sa volonté d'impulser de manière décisive le développement de la petite enfance : « l'État a choisi d'investir sur le capital enfant en vue d'en assurer une relève de qualité. Le développement de la petite enfance au Sénégal n'est point une action 
d'ordre social mais une action macro-économique qui se place au cœur du développement à travers la valorisation des ressources humaines ${ }^{3}$. Cette option du nouveau régime place la petite enfance au nombre des priorités de la politique éducative ; le Sénégal amorce un virage important dans la conceptualisation des politiques et l'implantation des structures et des services éducatifs destinés à l'éducation et à la protection de la petite enfance. Sur une idée du président de la République, une structure nouvelle s’invite dans le paysage éducatif sénégalais : le modèle présidentiel de la case des tout-petits (CTP). Dès 2001, le pilotage institutionnel du secteur de la petite enfance est confié non plus à une direction nationale mais tour à tour à un ministère délégué chargé de la petite enfance, puis à un ministère autonome, le ministère de la famille et de la petite enfance (MFPE), et enfin à l'Agence nationale de la case des tout-petits (ANCTP), service rattaché directement à la présidence. Cette structure que l'on croise aux détours des villes et des campagnes est-elle différente des structures existantes?

\section{Pourquoi la case des tout-petits ?}

Au Sénégal, les tout-petits (0-8 ans) représentaient, en 2000, 26,2 \% de la population totale (ANCTP, 2004). D’une manière générale, la situation de ces enfants est préoccupante. Le taux de mortalité des enfants de moins de 5 ans reste élevé (119 pour 1000 naissances en 2005) comparé aux normes mondiales, mais faible par rapport aux pays de la sous-région (Unesco, 2009). La malnutrition, qui atteint 22,7 \% des enfants âgés de moins de 5 ans $^{4}$, est responsable de $30 \%$ des cas d'hospitalisation des enfants.

L'état sanitaire et social des enfants au Sénégal est peu favorable. Des carences alimentaires dégradent l'état de santé des populations, les conditions d'hygiène et d'assainissement sont précaires. En milieu urbain en particulier, les disparités entre quartiers riches et pauvres s'accentuent. Le travail des enfants est encore une pratique courante $(37 \%)^{5}$.

La protection des enfants, malgré des efforts réels, reste très préoccupante. L'on assiste à un affaiblissement, à une relative désorganisation de la cellule familiale du fait d'une urbanisation sauvage due à l'exode rural. La cellule familiale assume difficilement l'éducation et la protection des jeunes enfants. La violation des droits des enfants est manifeste sous différentes formes : violences familiales, abus, exploitation par le travail et la mendicité, un fléau de la société sénégalaise. Les ressources financières allouées à la petite enfance n'assurent pas une bonne couverture sociale éducative (ANCTP, 2004). La croissance rapide de la population exerce de fortes pressions sur le système éducatif. La proportion

\footnotetext{
3. Document d'orientation stratégique du ministère de la famille et de la petite enfance (MFPE, 2002).

4. La moyenne de l'Afrique subsaharienne est de 29,9\% (rapport UNESCO, 2009).

5. Unesco, 2009.
} 
de ménages vivant au-dessous du seuil de pauvreté a atteint $58 \%$ en $1996^{6}$. Sous l'égide de la Banque mondiale, le Sénégal a élaboré un Document de stratégie de réduction de la pauvreté (DSRP) dont l'objectif prioritaire est de sortir de la pauvreté par la croissance. L'un des axes majeurs de cette stratégie est le développement de l'éducation et de la formation.

Face à cette situation, les nouvelles autorités font de la prise en charge de la petite enfance une priorité de développement. Une nouvelle "politique nationale pour le développement intégré de la petite enfance (PNDIPE) » est élaborée, puis adoptée en 2006. Elle vise à élargir l'accès aux structures de la petite enfance et à s'attaquer ainsi aux inégalités, afin de garantir la protection de la cellule familiale, premier lieu d'éducation. La mise en ouvre de cette politique donne naissance au programme de la case des tout-petits dont l'ANCTP assure le pilotage.

\section{La nouvelle politique de développement intégré de la petite enfance}

Dans le but d'aider les États à développer et à améliorer les services de la petite enfance, l'UNESCO a lancé avec l'OCDE un projet conjoint de révision des politiques de la petite enfance. Le Sénégal a participé à cette activité en $2007^{7}$, examen qui lui a permis de faire le bilan de sa politique de développement intégré de la petite enfance (DIPE) mais également de valider le modèle de la CTP dont le processus est en cours.

La politique DIPE vise tout d'abord la reconnaissance et la promotion des droits de l'enfant ; ce principe affirme explicitement le droit des enfants à être protégés. Un autre principe, l'approche holistique, met l'accent sur l'ensemble des besoins de l'enfant qui sont à promouvoir. Au lieu d'une intervention fragmentée, éclatée en faveur de la petite enfance, il prévoit une approche globale, coordonnée, s'inscrivant dans un continuum allant de la période prénatale à l'accès à l'école élémentaire. Est également adopté le principe d'une approche communautaire du développement de la petite enfance, dans laquelle l'implication active des parents et des communautés locales peut garantir la réussite d'une prise en charge intégrée. Le dernier principe, et pas des moindres, souligne l'ancrage culturel de cette politique. Le programme entend s'enraciner dans les valeurs du milieu tout en y intégrant les acquis scientifiques.

Les stratégies développées reposent d'une part sur une politique de communication en direction des populations bénéficiaires, des acteurs et des partenaires institutionnels et financiers pour les sensibiliser à l'importance du

6. ME/CREA (2004), Analyse du secteur de l'éducation.

7. Le Sénégal, deuxième pays africain à bénéficier de cette activité, a été sélectionné pour la deuxième phase de ce projet. Membre de l'équipe de la revue, nous avons participé aux différentes activités liées à cet exercice. Le présent article développe quelques unes de ses conclusions. 
développement de la petite enfance, et d'autre part sur le développement d'une dynamique partenariale large autour des intérêts du jeune enfant et passant par la mise en place de comités multisectoriels (coopération bilatérale, ONG nationales et internationales, etc.).

\section{Signification et fonctionnement des cases des tout-petits}

La «case », selon le dictionnaire Robert, désigne "une habitation traditionnelle généralement construite en matériaux légers dans certaines civilisations des pays tropicaux ». Le choix de ce concept pour désigner les nouvelles structures d'éducation et de protection de la petite enfance n'est point fortuit.

La case en Afrique, souvent construite à l'aide de matériaux naturels, est un symbole de la civilisation ancestrale. Elle traduit un mode de vie, une manière d'être et de penser. Elle symbolise un attachement à des valeurs négroafricaines. La case, espace de vie, espace socialisé, lieu éducatif par excellence était le point de départ de l'apprentissage de la vie d'homme. C'est dans la case de sa mère que l'enfant vit l'expérience de la dyade mère-enfant qui évolue, avec le sevrage, vers l'ouverture au groupe familial. L'investissement de l'enfant par les autres membres de la famille et le début de sa participation aux activités sociales du groupe, à travers lesquelles s'effectue la transmission des normes et valeurs ont lieu dans la case. Par ailleurs, durant les cérémonies rituelles de la circoncision, le séjour dans la " case d'homme » marquait le passage à la puberté, à la classe d'âge supérieure, ce qui correspond à une nouvelle étape d'insertion sociale, d'intégration de l'individu à sa communauté. C'est aussi devant la case de la grand-mère ou du grand-père que se déroulaient les veillées africaines faites de contes et de longues palabres.

La CTP est une structure communautaire de prise en charge des enfants âgés de 0 à 6 ans. Le président A. Wade en a lui-même conçu la maquette et manifeste un engagement personnel fort en sa faveur, à travers un ambitieux programme d'implantation de 28000 cases sur l'ensemble du territoire d'ici 2010. La CTP, présentée comme modèle à promouvoir, a pour objectif « d'assurer à tous les enfants sénégalais, de la conception à 6 ans, issus en priorité de milieux défavorisés, l'accès à des services adéquats et intégrés, selon une approche participative pour que les besoins de chacun d'entre eux soient pris en charge... dans un environnement sain » (ANCTP, 2004).

C'est un modèle de référence devant servir à plus ou moins long terme de système d'offre harmonisé pour le développement intégré de la petite enfance. Elle fait partie des structures communautaires car elle est gérée par les populations elles mêmes. Mais, à la différence des centres communautaires classiques, l'État prend directement en charge la construction des cases, les salaires du personnel d'encadrement ainsi que les dépenses de fonctionnement 
et d'équipement ${ }^{8}$. Son implantation privilégie les zones rurales et péri-urbaines pour cibler davantage les enfants vulnérables issus de milieux défavorisés. La CTP représente $19 \%$ des structures de DIPE. En 2007, parmi les 180 cases construites et fonctionnelles sur l'ensemble du territoire, 106 (soit $59 \%$ ) se trouvaient en zone rurale (Unesco, 2009).

Au plan architectural, la CTP est une structure de forme hexagonale abritant deux salles, dont l'une est destinée aux activités éducatives et l'autre à l'éducation parentale. On trouve dans cet espace une cuisine, une infirmerie, un bureau du personnel d'encadrement, des toilettes, un magasin de stockage des vivres et une cour aménagée abritant les jeux, les activités de plein air et les activités de micro-projets (maraîchage, poulailler, teinture, etc.). La barre oblique qui accompagne la pente de la toiture est le signe distinctif de la CTP : elle symbolise l'enracinement de l'enfant dans sa culture et son ouverture à la civilisation de l'universel. L'architecture d'une deuxième génération des cases a été améliorée grâce aux apports de la $\mathrm{JICA}^{9}$ pour faciliter la conduite des activités de l'approche intégrée.

La construction ${ }^{10}$ des cases évolue mais à un rythme lent, la situation économique n'étant pas favorable. Des cases sous abris provisoires prolifèrent, surtout en milieu rural. En 2007, sur les 338 cases construites et fonctionnelles, 158 fonctionnaient dans des abris provisoires dont $90,5 \%$ se trouvaient en zone rurale (DPRE, 2008).

\section{Localisation et financement}

Au Sénégal, l'offre préscolaire est segmentée selon le milieu géographique et selon le secteur. C'est un phénomène majoritairement urbain : $62 \%$ des structures sont implantées en milieu urbain et $38 \%$ en milieu rural. Le secteur privé domine en milieu urbain, notamment dans la région de Dakar. Le secteur communautaire est très présent en milieu rural où les cases communautaires et les CTP représentent chacune un tiers des structures présentes, surtout dans la région de Thiès (Unesco, 2009). L'État apporte un soutien accru à l'implantation des CTP. Cependant, l'implantation des CTP obéit à une logique de la demande, demande émanant des communautés locales. Un tel processus ne favorise pas l'accès des CTP aux plus pauvres. Toutefois, les disparités entre zone rurale et zone urbaine tendent à se résorber en ce qui concerne les effectifs et les structures, grâce à la politique d'implantation des CTP qui milite en faveur de la zone rurale $(35,8 \%$ contre $8 \%$ en zone urbaine). Le taux brut de préscolarisation est en hausse régulière : 6,5\% en 2005 (8,8\% en 2008), $10 \%$ étaient projetés en 2010 .

8. Le personnel d'appui est payé par la communauté avec la participation financière des familles.

9. Japan Cooperation Agency (Agence japonaise de coopération).

10. 338 cases construites dont 214 en milieu rural. 
La prise en charge dans les CTP n'est pas gratuite ${ }^{11}$. Il est demandé aux parents une participation financière dont le montant varie selon le type de structure, le secteur et l'environnement économique. Cette cotisation parentale peut être un frein à l'élargissement de l'accès et à la démocratisation de l'éducation, nuire à la politique d'équité du programme. Il est à remarquer que les CTP appliquent des tarifs plus bas que les autres structures du secteur formel. $\mathrm{Du}$ reste, cette participation financière est de portée symbolique : elle permet aux familles de travailler en synergie autour d'un bien commun appartenant à la communauté et qu'elle a le devoir de préserver. C'est une manière de combattre la mentalité de la "main tendue », responsable de l'échec d'un certains nombre de projets en Afrique.

$\mathrm{Au}$ niveau du secteur formel, seules les CTP et un certain nombre de cases communautaires (CC) offrent un service aux enfants de moins de trois ans ${ }^{12}$. Selon le rapport de la revue de l'UNESCO, deux tiers seulement des CTP développent des activités relatives au couple mère-enfant et à la femme enceinte. La prise en charge des enfants à besoin spéciaux n'est pas encore effective dans les CTP, ce type d'éducation demeurant du reste marginal au Sénégal.

\section{Les conditions d'apprentissage des enfants}

Comparée aux autres structures, la CTP est mieux équipée (mobilier, équipement de plein air, matériel pédagogique, de cuisine, d'infirmerie, etc.). Il existe un déséquilibre entre structures (CTP, écoles maternelles, cases communautaires) en ce qui concerne la dotation en matériel mais aussi des différences d'une région à une autre. Dans les CTP, l'effectif est limité à vingt enfants, ce qui permet un bon déroulement de l'approche intégrée ; ce taux d'encadrement est inférieur à la moyenne nationale qui est de 27 élèves par section. L'ANCTP a acquis, en partenariat avec un éditeur français, un important lot de matériel pédagogique. L'introduction d'un matériel de fabrication locale se fait timidement.

Les animateurs polyvalents et les volontaires de la petite enfance sont en charge des activités. Des mères assistantes participent au déroulement des activités tout en veillant à la propreté et à l'entretien des locaux. Une grandmère ou un grand-père initie à l'éducation morale et culturelle. Un ou deux maîtres coraniques interviennent pour la formation religieuse. Les animateurs polyvalents sont recrutés sur la base du brevet de fin d'études moyennes ou du baccalauréat. L'ANCTP leur dispense une formation spécifique de deux mois, centrée sur les objectifs de la PNDIPE et l'approche holistique. Depuis peu, elle est complétée par une formation de six mois dans les écoles de formation des

11. Le montant de la participation financière des parents est compris entre $200 \mathrm{Fcfa}$ et $2000 \mathrm{Fcfa}$.

12. Il existe des crèches privées mais leur nombre est pour l'instant très restreint. 
instituteurs (EFI), ce qui leur confère un statut professionnel plus favorable, volontaire de l'éducation de la petite enfance.

Des inspecteurs de l'éducation préscolaires, d'un niveau de formation supérieur sont nommés coordonnateurs des antennes régionales de l'ANCTP. Ils assurent la supervision des structures DIPE, la formation et l'encadrement des personnels, ainsi que le suivi et l'encadrement des comités de gestion des CTP. $\mathrm{Au}$ niveau départemental, les coordonnateurs sont accompagnés par des enseignants appelés " points focaux ». L'action de suivi et d'encadrement de ces derniers est renforcée par les membres du collectif départemental des encadreurs de la petite enfance (CODEPE).

\section{LES PROGRAMMES}

Les programmes de la CTP développent une approche incluant l'éducation, la santé et la nutrition. La CTP propose une offre diversifiée de services qui se déclinent sur le plan éducatif en activités d'éveil et de socialisation et en activités culturelles (transmission des valeurs traditionnelles).

Sur le plan sanitaire, l'intervention est axée sur les soins et le dépistage de carences et de troubles chez les enfants, la formation et l'information des mères. Ces activités sont menées par les animateurs polyvalents, en collaboration avec les agents de santé.

Sur le plan nutritionnel, les programmes veulent améliorer la nutrition dans la petite enfance, en corrigeant certaines carences alimentaires très fréquentes en Afrique, surtout en milieu rural, car améliorer la nutrition et réduire la faim dès la petite enfance sont indispensables pour atteindre les Objectifs du millénaire pour le développement (OMD). Ainsi, des repas équilibrés sont préparés par les mères assistantes et servis aux enfants. Des denrées alimentaires (lait, farine enrichie) sont distribués aux couples mères-enfants. Les animateurs polyvalents sont formés à identifier les besoins nutritionnels de l'enfant de 0 à 6 ans, de la femme enceinte et de la mère allaitante.

Outre ces activités principales, d'autres activités complémentaires telles la familiarisation à l'informatique, le micro-projet, le renforcement des capacités des parents, familles et communautés, contribuent à renforcer le volet social et environnemental.

Comme on peut le constater, ces programmes sont complexes parce qu'ils veulent toucher l'enfant dans sa globalité et dans son environnement. Ils ont des exigences énormes en ressources humaines, matérielles et financières à satisfaire par des stratégies basées sur une dynamique partenariale et une bonne politique de communication. La diffusion et l'harmonisation de l'approche holistique et intégrée est un objectif majeur qui conditionne le succès de la politique DIPE, de même que la pérennité de la CTP. L'approche intégrée est prise en compte dans le nouveau curriculum mais sans une formation initiale adéquate, est-elle bien comprise, bien maîtrisée? 
Dans les structures DIPE, le médium d'enseignement le plus utilisé est le français (82\%), suivi de l'arabe $(11 \%)$ et des langues nationales (7\%). À ce niveau également, l'adéquation de la formation aux exigences de la NPDIPE est impérieuse.

La gestion de la CTP est une gestion à la base. Pour ce faire, l'ANCTP a mis en place une organisation spécifique pour la gestion de la petite enfance au niveau national, régional et, au niveau des structures de prise en charge (CTP, garderies communautaires), un système local de gestion communautaire impliquant toutes les couches de la société. Cette gestion décentralisée rencontre de nombreuses difficultés parmi lesquelles on peut citer le nombre infime de comités de gestion qui fonctionnent, l'absence de formation pour les membres des comités de gestion, la faiblesse de la participation des communautés qui ne favorise pas une gestion consensuelle et rationnelle des ressources.

Le programme des CTP est relativement jeune, les premières cases n'ayant véritablement fonctionné qu'à partir de $2002^{13}$. C'est donc avec un faible recul que l'on peut l'évaluer. Il jouit d'un atout majeur : l'engagement du président de la République qui a joué en faveur de la popularité du modèle et de sa diffusion, mais également dans l'implication des divers acteurs et partenaires. Après quelques années seulement et au regard des réalisations de ce programme, quel bilan peut-on dresser de ses acquis en matière d'équité, de qualité et de diversité de l'accueil ?

L'implantation de la CTP en priorité dans le milieu rural est une innovation de taille, car l'éducation préscolaire est un phénomène urbain. D’où l'accueil favorable et l'adhésion des populations rurales à ce projet auquel elles participent, qui engage leur responsabilité, valorise leurs savoirs et leurs savoirfaire. Cependant, dans certains cas, cette adhésion forte a pu desservir le programme en entraînant des confusions voire des dérives dans sa mise en œuvre ${ }^{14}$.

\section{Accès et équité}

Il faut souligner les avancées en matière d'accessibilité à l'éducation. Les structures CTP connaissent une évolution régulière - un facteur déterminant dans la démocratisation de l'accès - avec le développement de la capacité d'accueil du secteur. Mais l'implantation des structures doit se faire avec plus de cohérence pour réduire la pauvreté, surtout dans les zones où la prévalence de la pauvreté est importante. La part du communautaire était en hausse de 13,5\% en 2005 et de $29 \%$ en 2008, tandis que celle du privé avait tendance à diminuer aussi bien pour les structures que pour les effectifs (DPRE, 2008) ; ce résultat est la conséquence d'une politique dynamique de préscolarisation à laquelle la

\footnotetext{
13. Pour une information complète sur l'implantation et le démarrage des CTP, voir S. Rayna (2002).

14. La politique politicienne s'est souvent invitée dans ces projets dédiés à l'enfance : des responsables locaux en quête de promotion politique ont tendance à intervenir au niveau du programme CTP (implantation, équipement, choix du personnel).
} 
CTP participe et qui donne priorité aux zones défavorisées. La part des filles est en léger progrès dans les structures ${ }^{15}$ et dans certaines régions.

Cependant, si l'on veut donner aux enfants une éducation équitable il faut éliminer les disparités à tous les niveaux ; élargir l'accès aux CTP en y aménageant des formules crèche pour l'accueil des moins de trois ans; trouver des solutions pour réduire le coût prohibitif de construction de la case ; en ce qui concerne l'implantation des cases, suivre une logique de l'offre pour garantir l'équité des services.

\section{Qualité de l'éducation}

Les CTP sont les structures les plus avancées dans l'application de l'approche holistique et intégrée. La consolidation du volet santé-nutrition souffre d'un manque de coordination multisectorielle. Pour l'essentiel, les conditions d'apprentissage sont satisfaisantes dans les CTP, le taux d'encadrement est raisonnable. L'espace pédagogique est amélioré tout en respectant le prototype, et réaménagé en tenant compte des exigences de l'approche intégrée. Le nouveau curriculum comporte une entrée par les compétences, sa mise en œuvre est planifiée, des guides et manuels sont édités et mis à la disposition des centres de développement intégré de la petite enfance (CDIPE). Un curriculum spécifique pour l'éducation et la protection des enfants de moins de trois ans et ceux de l'éducation spéciale est en cours d'élaboration. Le choix de la langue d'enseignement n'est pas encore tranché, le français est la langue dominante et les langues nationales sont à la traîne. Il reste à définir une politique claire et réaliste qui fixerait la place des langues nationales dans le système éducatif sénégalais en général et dans les programmes DIPE en particulier.

Au niveau de la formation, l'organisation de classes spéciales pour le préscolaire au niveau des EFI est une conquête qui mérite d'être soulignée. La formation n'est toutefois pas en cohérence avec les exigences de l'approche holistique intégrée. Au total, les maux dont souffre la formation sont : la polyvalence perçue comme une perte des acquis par les acteurs sur le terrain, la durée trop courte de la formation initiale et le niveau de qualification faible pour la complexité de l'intervention éducative. La formation du personnel, le renforcement des capacités des mères-assistantes, grand-mères, la formation en gestion des communautés sont autant de choix à faire pour améliorer la qualité de l'éducation.

La part du budget de fonctionnement est insuffisante et le financement du programme doit être diversifié en encourageant les contributions volontaires locales, nationales et internationales. 
De l'ouverture de la case témoin de Déni Biram Ndao à ce jour, de nombreux défis ont été relevés mais il reste encore un long chemin à parcourir pour améliorer ce programme et le rendre plus efficace. La case des tout-petits sort de l'enfance : elle se développe au plan quantitatif en se multipliant à grande échelle et au plan qualitatif en participant à la diffusion, à l'harmonisation de l'approche intégrée, et à l'innovation pour le développement et l'épanouissement des jeunes enfants. La case des tout-petits, un îlot de verdure au milieu du désert? Il est clair que de l'issue des innovations qui ont cours actuellement au niveau des CTP dépend l'avenir du secteur. L'enjeu de la CTP est d'offrir des perspectives intéressantes sur le plan de l'harmonisation des pratiques, de la construction de nouvelles professionnalités et du maintien des standards de qualité et d'équité. La CTP, une utopie ? Plutôt un ambitieux projet réalisable à force de réalisme et de choix éclairés à la dimension de la conviction de ses acteurs.

\section{BIBLIOGRAPHIE}

ANCTP (2006) : La case des tout-petits, une expérience novatrice de prise en charge de la petite enfance, Dakar.

ANCTP (2004) : Politique nationale de développement intégré de la petite enfance au Sénégal, Dakar.

DPRE-PDEF (2008) : Rapport national sur la situation de l'éducation, ME/Dakar.

RAYNA S. (2002) : "La mise en œuvre de la politique intégrée de la petite enfance au Sénégal », Unesco, Série sur la petite enfance, $\mathrm{n}^{\circ} 2$, septembre.

UNESCO (2009) : «Rapport de revue de la politique d'éducation et de protection de la petite enfance au Sénégal ", Série sur la politique de développement de la petite enfance et de la famille $\mathrm{n}^{\circ} 15$. 
